Quim. Nova, Vol. 34, No. 8, 1328-1333, 2011

\title{
AVALIAÇÃO GEOQUÍMICA DE ÓLEOS BRASILEIROS COM ÊNFASE NOS HIDROCARBONETOS AROMÁTICOS
}

\author{
Jean Romei Heckmann* e Luiz Landau \\ 21941-909 Rio de Janeiro - RJ, Brasil \\ Félix Thadeu Teixeira Gonçalves \\ Vale Óleo e Gás, 20020-900 Rio de Janeiro - RJ, Brasil \\ Ricardo Pereira e Débora de Almeida Azevedo \\ Instituto de Química, Universidade Federal do Rio de Janeiro, 21941-909 Rio de Janeiro - RJ, Brasil
}

COPPE - Instituto Alberto Luiz Coimbra de Pós-graduação e Pesquisa de Engenharia, Universidade Federal do Rio de Janeiro,

Recebido em 18/10/10; aceito em 15/3/10; publicado na web em 5/5/11

\begin{abstract}
GEOCHEMICAL EVALUATION OF BRAZILIAN OILS WITH EMPHASIS ON AROMATIC HYDROCARBONS. Aromatic compounds are still poorly evaluated in geochemical studies of Brazilian oil samples. For this reason, the objective of this work was to analyze and evaluate 26 oil samples from four Brazilian sedimentary basins. Aromatic hydrocarbon fractions have been analyzed by gas chromatography coupled to mass spectrometry. The results were also compared with previously evaluated saturated hydrocarbon data. Maturation parameters obtained using the 4- and 1-methyl-dibenzothiophenes and trimethyl-dibenzothiophenes led to the best results because a better grouping was observed. For this correlation, the samples were grouped by maturity degree enabling the routine use of these parameters to evaluate oil maturity levels.
\end{abstract}

Keywords: biomarkers; aromatic hydrocarbon; maturity evolution.

\section{INTRODUÇÃO}

O petróleo é uma mistura complexa composta principalmente de hidrocarbonetos saturados, hidrocarbonetos aromáticos, resinas e asfaltenos. O estudo de suas características geoquímicas por meio da análise de biomarcadores é indispensável para a indústria petrolífera. Neste contexto, é de grande importância a avaliação de parâmetros relacionados com a geração, migração e acumulação de petróleo, a fácies estratigráfica da rocha geradora, bem como as profundidades nas quais prevalecem as condições diagenéticas ótimas para a formação do petróleo. ${ }^{1,2}$

Diversas informações podem ser obtidas a partir dos biomarcadores. O grau de evolução térmica, por exemplo, pode ser monitorado através de diferentes razões de concentrações relativas entre o isômero mais estável e o menos estável. ${ }^{2}$ A aromatização de compostos e a degradação térmica, onde compostos menos estáveis tendem a desaparecer em relação aos mais estáveis, permite estabelecer o grau de evolução de óleos e da matéria orgânica em sedimentos. ${ }^{2}$ Também é possível o estabelecimento de correlações óleo/óleo e óleo/rocha geradora. Óleos de origens diferentes apresentam composições geoquímicas de biomarcadores distintas, ou seja, óleos de origem marinha e/ou terrestre produzem petróleos de características diferenciadas. Em óleos de origem marinha, a matéria orgânica é proveniente basicamente de plantas aquáticas (fitoplâncton) que conferem uma composição química totalmente diferente dos petróleos de origem terrestre, onde é expressiva a contribuição de matéria orgânica proveniente de vegetais superiores. A correlação óleo/óleo requer parâmetros comumente não alterados por processos geoquímicos, tais como biodegradação, evolução e fracionamento evaporativo. ${ }^{2}$

Os hidrocarbonetos aromáticos estão entre os maiores constituintes da matéria orgânica sedimentar podendo ser utilizados como

\footnotetext{
*e-mail: jean.heckmann@hotmail.com
}

biomarcadores, assim como os hidrocarbonetos saturados, esteroides e hopanos. No entanto, hidrocarbonetos aromáticos representados por alquilbenzenos, alquilnaftalenos, alquilfenantrenos e compostos aromáticos sulfurados não são formalmente considerados como biomarcadores, por terem sofrido alterações significativas na estrutura básica original em relação às suas moléculas precursoras. São, portanto, provenientes de biomarcadores, mas não guardam uma relação inequívoca com os compostos que os originaram. Ainda assim é possível aplicá-los na determinação de diversos parâmetros geoquímicos relacionados como, por exemplo, a origem da matéria orgânica, o ambiente de deposição e a evolução térmica. ${ }^{3,4}$ Alquilbenzenos, alquilnaftalenos e alquilfenantrenos podem ser utilizados como indicadores de maturidade térmica para a matéria orgânica. ${ }^{3,4}$ Estudos realizados em geoquímica orgânica também demonstraram que os biomarcadores aromáticos podem fornecer informações valiosas com respeito à contribuição da matéria orgânica e em correlações óleo/ óleo e óleo/rocha geradora. ${ }^{2,5}$ A Tabela 1 apresenta alguns parâmetros geoquímicos baseados na análise de compostos aromáticos.

Os hidrocarbonetos aromáticos, como o fenantreno e os seus alquilderivados, podem ser encontrados em petróleos e em matéria orgânica sedimentar, sendo derivados de esteroides e triterpenoides. ${ }^{6}$ Devido a grande ocorrência de hidrocarbonetos aromáticos com esqueleto não isoprenoidal em sedimentos, acredita-se que estes possam ser produtos de reações sedimentares. ${ }^{7}$ A ocorrência dos alquilfenantrenos tem sido atribuída geralmente ao aporte de matéria orgânica terrestre..$^{8,9}$

As razões de metil-naftaleno (RMN) e etil-naftaleno (REN) são indicadores úteis para evolução térmica. ${ }^{2,6,7,10} \mathrm{O}$ naftaleno e seus derivados alquilados estão ausentes ou em baixa concentração em sedimentos pouco evoluídos. No entanto, com o incremento da evolução térmica há um enriquecimento do isômero termodinamicamente mais estável, que corresponde à estrutura alquilada na posição $\beta .{ }^{10}$ 
Tabela 1. Parâmetros geoquímicos analisados para hidrocarbonetos aromáticos $6,8,11,12$

\begin{tabular}{lc}
\hline $\operatorname{Siglas}^{(*)}$ & Parâmetros \\
\hline RMN & $2 \mathrm{MN} / 1 \mathrm{MN}$ \\
REN & $2 \mathrm{EN} / 1 \mathrm{EN}$ \\
RDMN & $(2,6-\mathrm{DMN}+2,7-\mathrm{DMN}) / 1,5-\mathrm{DMN}$ \\
RTMN & $2,3,6-\mathrm{TMN} /(1,4,6-\mathrm{TMN}+1,3,5-\mathrm{TMN})$ \\
RMF & $2-\mathrm{MF} / 1-\mathrm{MF}$ \\
IMF & 4-MDBT/F \\
RMD & $4-\mathrm{MDBT} / 1-\mathrm{MDBT}$ \\
RMDBT4/1 & 2,4-DMDBT/1,4-DMDBT \\
RMDBT2,4/1,4 & $4,6-\mathrm{M}+3-\mathrm{MF}) /(\mathrm{F}+1-\mathrm{MF}+9-\mathrm{MF})$ \\
RMDBT4,6/1,4 & TMDBT (substância 38)/TMDBT (substância 40) \\
RTMDBT(38/40)
\end{tabular}

(*): RMN = razão 2-metil-naftaleno/1-metil-naftaleno; $\mathrm{REN}=$ razão 2-etil-naftaleno/1-etil-naftaleno; RDMN = razão (2,6-dimetil-naftaleno + 2,7-dimetil-naftaleno)/1,5-dimetil-naftaleno; RTMN = razão 2,3,6-trimetilnaftaleno/(1,4,6-trimetil-naftaleno+1,3,5-trimetil-naftaleno); RMF = razão 2-metil-fenantreno/1-metil-fenantreno; IMF = índice de metil-fenantrenos; 3-MF = 3-metil-fenantreno; $\mathrm{F}$ = fenantreno; 9-MF = 9-metil-fenantreno; RMD = razão 4-metil-dibenzotiofeno/fenantreno; RMDBT4/1 = razão 4-metildibenzotiofeno/1-metil-dibenzotiofeno; RDMDBT2,4/1,4 = razão 2,4-dimetildibenzotiofeno/1,4-dimetil-dibenzotiofeno; RDMDBT4,6/1,4 = razão 4,6-dimetil-dibenzotiofeno/1,4-dimetil-dibenzotiofeno; RTMDBT $(38 / 40)=$ razão trimetil-dibenzotiofeno (substância 38)/trimetil-dibenzotiofeno (substância 40).

A distribuição dos metil-fenantrenos (MF) depende da origem da matéria orgânica - marinha, lacustre ou terrestre. As amostras de origem marinha apresentam maior abundância de 9-metil-fenantreno (isômero $\alpha$ ) [9-MF] e baixa concentração de 3- e 2-MF ( $\beta$ ), enquanto que a presença de $1-\mathrm{MF}$ (isômero $\alpha$ ) e 2-MF (isômero $\beta$ ) pode estar associada ao aporte de matéria orgânica de origem terrestre. ${ }^{4}$ Diversas pesquisas foram realizadas quanto à utilidade dos derivados alquilados do fenantreno como parâmetros de evolução térmica, sendo que os mais estudados são o 1-, 2-, 3- e 9-MF. ${ }^{8}$ Em amostras de óleo pouco evoluídas termicamente, os isômeros 9-MF e 1-MF são os de maior abundância, sendo 9-MF o isômero predominante. Com o aumento do nível da evolução térmica, os isômeros 2-MF e 3-MF sofrem um incremento em suas concentrações, devido a maior estabilidade térmica do isômero $\beta$ sobre o isômero $\alpha{ }^{4,11}$

Os isômeros 2,6- e 2,7-dimetil-fenantreno, por outro lado, apresentam maior estabilidade e podem ser encontrados em maiores proporções em amostras com aporte de matéria orgânica de origem terrestre. Verifica-se que óleos marinhos e extratos de rocha geradora apresentam baixas concentrações destes compostos quando comparados com óleos de origem terrestre. ${ }^{4}$

Os compostos aromáticos sulfurados, como benzotiofenos e dibenzotiofenos, são constituintes comuns de óleos e matéria orgânica de rochas geradoras, também sendo ferramentas úteis para a avaliação geoquímica. ${ }^{12}$ Os benzotiofenos e dibenzotiofenos são abundantes em óleos maturos e foram propostos como indicadores de evolução térmica. Entretanto, estudos realizados sobre a ocorrência e evolução térmica de alquilbenzotiofenos e dibenzotiofenos em rochas geradoras de petróleo mostraram que as distribuições de benzotiofenos são úteis somente para interpretação de evolução térmica. ${ }^{8}$ As relações dos isômeros baseados em metil-, etil- e dimetil-dibenzotiofenos servem como parâmetros de evolução térmica de rochas geradoras, apresentando os melhores resultados em comparação aos dados obtidos por reflectância de vitrinitas. ${ }^{10}$
Em trabalhos relacionados à avaliação geoquímica de óleos brasileiros tem sido dada pouca ênfase aos hidrocarbonetos aromáticos, objetivo principal deste estudo. Muitos óleos brasileiros têm como característica valores elevados dos índices de aromáticos. Isto, aliado ao fato de que tais compostos foram pouco estudados, mostra a importância deste trabalho para a indústria do petróleo. Desta forma, foram avaliadas amostras selecionadas de óleos provenientes de diversas bacias sedimentares brasileiras (Camamu-Almada, Jequitinhonha, Sergipe-Alagoas e Santos), com ênfase na fração dos hidrocarbonetos aromáticos. Estes permitem obter melhores informações quanto à maturação, tendo maior resistência ao processo de biodegradação e contribuindo assim na identificação de famílias de petróleos.

\section{PARTE EXPERIMENTAL}

\section{Amostras}

As amostras de óleos avaliadas neste estudo são provenientes das bacias Camamu-Almada (CA), Jequitinhonha (JE), Sergipe (SE), Alagoas (AL) e Santos (SA). Foram selecionadas destas bacias um grupo de 26 amostras de óleo buscando diferentes níveis de evolução térmica e ausência de processo de biodegradação, a partir de dados relatados em trabalhos anteriores. ${ }^{13}$

\section{Fracionamento dos óleos}

Aproximadamente $100 \mathrm{mg}$ de cada amostra de óleo foram submetidos a fracionamento utilizando coluna cromatográfica de vidro (dimensões de 13 x $1 \mathrm{~cm}$ ) com 2,5 g de sílica gel neutra (0,063-0,200 mm, kieselgel 60, 70-200 mesh, Merck n 107734 - Rio de Janeiro) recém-ativada a $120{ }^{\circ} \mathrm{C}$ durante $12 \mathrm{~h}$, procedimento baseado em trabalhos anteriores. ${ }^{13,14}$

A fração dos hidrocarbonetos saturados foi eluída com 10 $\mathrm{mL}$ de $n$-hexano, enquanto as frações contendo hidrocarbonetos aromáticos e aquela contendo compostos polares foram eluídas com $10 \mathrm{~mL}$ de $n$-hexano:diclorometano (1:1, v:v) e com $10 \mathrm{~mL}$ de diclorometano:metanol (9:1, v:v), respectivamente. As soluções obtidas foram concentradas em evaporador rotatório e transferidas para frascos de $2 \mathrm{~mL}$ previamente pesados. A evaporação do solvente residual foi realizada sob fluxo de nitrogênio, havendo posterior pesagem de cada fração. ${ }^{13,14}$

\section{Cromatografia gasosa acoplada à espectrometria de massas (CG-EM)}

Foram injetados $2 \mu \mathrm{L}$ de cada uma das frações de hidrocarbonetos aromáticos em um cromatógrafo a gás Agilent Technologies 5890 acoplado a um espectrômetro de massas Agilent Technologies 5973, utilizando coluna capilar de sílica fundida recoberta com DB-5 (J\&W; $30 \mathrm{~m}$ x 0,25 mm d.i; d.f. = 0,25 mm). As condições de análise foram: temperatura do forno mantida a $40{ }^{\circ} \mathrm{C}$ por $5 \mathrm{~min}$, programação de temperatura de 40 a $300{ }^{\circ} \mathrm{C}, 4{ }^{\circ} \mathrm{C} / \mathrm{min}$, mantendo em isoterma por 10 min, e temperatura do injetor a $290^{\circ} \mathrm{C}$. Foi utilizado hélio como gás carreador, injeção com divisão de fluxo (1/5) e ionização por impacto de elétrons $(70 \mathrm{eV})$. As amostras foram analisadas por monitoramento seletivo de íons (MSI), utilizando para identificação os íons diagnósticos da Tabela 2. Algumas das amostras também foram analisadas no modo varredura linear na faixa de 50-560 Daltons para a obtenção dos espectros de massas dos analitos e confirmação de identificação dos mesmos.

Os picos detectados nos cromatogramas em MSI e referentes aos analitos de interesse foram integrados e as razões entre as áreas dos picos foram usadas na avaliação dos parâmetros geoquímicos analisados nos óleos estudados. 
Tabela 2. Íons diagnósticos $(\mathrm{m} / \mathrm{z})$ utilizados na identificação de compostos aromáticos por monitoramento seletivo de íons ${ }^{18}$

\begin{tabular}{lc}
\hline Nome do composto & Íons $(\mathrm{m} / \mathrm{z})$ \\
\hline Naftaleno & 128 \\
Metil-naftalenos & 142 \\
Dimetil-naftalenos & 156 \\
Trimetil-naftalenos & 170 \\
Tetrametil-naftalenos & 184 \\
Fenantreno & 178 \\
Metil-fenantrenos & 192 \\
Metil-dibenzotiofenos & 198 \\
Dimetil-dibenzotiofenos & 212 \\
Trimetil-dibenzotiofenos & 226
\end{tabular}

Os compostos foram identificados por comparação dos espectros de massas obtidos com os existentes na literatura, bibliotecas eletrônicas Wiley 275 e Nist Mass Spectral Database (Nist, versão 2.0, 2002), tempo de retenção, ordem de eluição, bem como pelos perfis de distribuição típicos das classes e comparadas com amostra de óleo utilizada como referência, assim como dados da literatura.

As razões de esteranos e de pristano/fitano utilizadas neste estudo foram obtidas de trabalhos anteriores realizados pelo mesmo grupo. ${ }^{13,14}$ Resumidamente, as frações dos hidrocarbonetos saturados para as mesmas amostras de óleo foram analisadas por cromatografia gasosa acoplada à espectrometria de massas utilizando monitoramento seletivo de íons.

\section{RESULTADOS E DISCUSSÃO}

Na Tabela 3 são apresentados os valores de parâmetros geoquímicos selecionados e calculados a partir das razões das áreas obtidas por CG/EM-MSI dos hidrocarbonetos aromáticos e saturados que permitem caracterizar as amostras dos óleos. Na Figura 1 são mostrados os cromatogramas de massas dos alquil-dibenzotiofenos identificados para a amostra CA-99, enquanto nas Figuras 1S e 2S (material suplementar) são apresentados os cromatogramas de massas do naftaleno e os seus derivados alquilados para a amostra JE-371 e os cromatogramas de massas do fenantreno e os seus alquilderivados para a amostra SE-2, respectivamente.

Todos os compostos identificados estão relacionados na Tabela 4. A numeração segue a apresentada nos cromatogramas das Figuras $1,1 \mathrm{~S}$ e $2 \mathrm{~S}$ (material suplementar).

\section{Ambiente deposicional e origem da matéria orgânica}

Diversos parâmetros para caracterização geoquímica do ambiente deposicional a partir da análise dos hidrocarbonetos aromáticos têm sido relatados. Um exemplo é a razão 4-metil-dibenzotiofeno/fenantreno (RMD). ${ }^{7,10,15,16}$ Neste estudo, para avaliar a origem da matéria orgânica, foi utilizado o gráfico entre a razão pristano/fitano $(\mathrm{Pr} / \mathrm{Fi})$ contra a razão RMD.

Alguns autores sugerem que os valores dos parâmetros RMD < 1 e $\mathrm{Pr} / \mathrm{Fi}<1$ indicam ambiente deposicional com caráter lacustre, enquanto as razões RMD $<1$ e $\mathrm{Pr} / \mathrm{Fi}$ entre 1 a 3 caracterizam ambiente deposicional com caráter marinho. ${ }^{2,17}$ Resultados semelhantes foram obtidos em outros trabalhos, quando foi verificado que estes parâmetros são uma ferramenta útil para caracterização do ambiente deposicional. $^{2}$
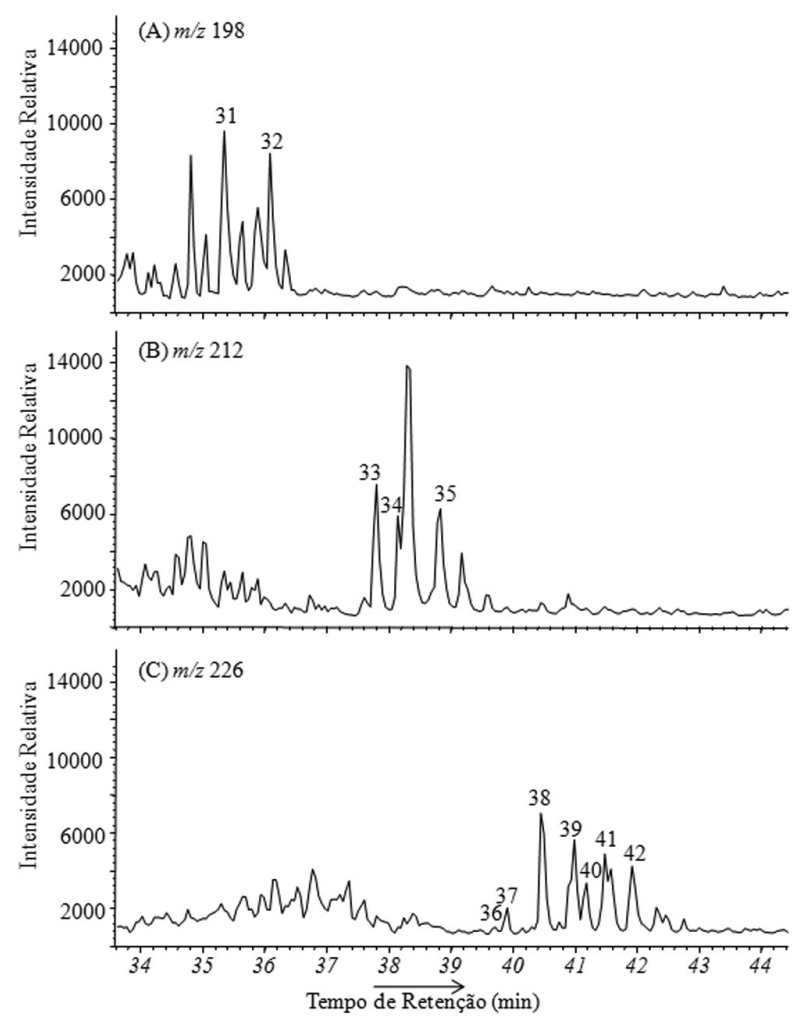

Figura 1. Cromatogramas de massas dos íons: (A) $\mathrm{m} / \mathrm{z}, 198$, característico dos metil-dibenzotiofenos, $(B) \mathrm{m} / \mathrm{z}, 212$, característico dos dimetil-dibenzotiofenos $e(C) \mathrm{m} / z, 226$, característico dos trimetil-dibenzotiofenos, demonstrando a distribuição destes, para a amostra CA-99. A identificação dos picos é apresentada na Tabela 4

Acredita-se que a variabilidade das razões Pr/Fi e RMD seja resultante das condições sulfato redutoras durante o processo deposicional, principalmente pela presença de compostos como ácido sulfídrico $\left(\mathrm{H}_{2} \mathrm{~S}\right)$ e por polissulfurados $\left(\mathrm{H}_{2} \mathrm{~S}_{n}\right)$ interagindo com a matéria orgânica. Entretanto, para que as reações sulfato redutoras sejam abundantes, os sedimentos devem estar em condições anóxicas considerando que as bactérias sulfato redutoras ocorrem obrigatoriamente em condições anaeróbicas. Deve haver, portanto, quantidades significativamente altas do íon $\mathrm{SO}_{4}^{2-}$ e também baixa concentração de compostos redutores metálicos. Baseado neste princípio, as razões Pr/Fi e RMD promovem uma base para a classificação de ambientes deposicionais, conforme se verifica nos resultados de trabalhos anteriores. ${ }^{2,16}$

Utilizando-se estes parâmetros de origem, somente conseguiuse classificar quanto à origem as amostras da Bacia de Santos, onde foi verificado que a maioria apresentou parâmetros classificando-as como de origem marinha. Tais resultados foram concordantes com aqueles encontrados em trabalhos anteriores a partir da análise de hidrocarbonetos saturados para as mesmas amostras. ${ }^{13}$ Para as demais amostras, estes parâmetros não foram conclusivos, isto é, as amostras poderiam ser de origem marinha ou lacustre.

\section{Evolução térmica}

Entre os parâmetros de evolução térmica que têm sido propostos para os hidrocarbonetos aromáticos, podem ser citados o índice de metil-fenantrenos (IMF) e a razão de metil-naftalenos (RMN)., 2,6,10 Neste estudo, os valores de diversas razões entre as áreas de picos correspondentes a substâncias ou a grupos de substâncias selecionadas foram empregados para avaliar e inferir a evolução térmica a partir da estabilidade térmica de compostos aromáticos sulfurados como, 
Tabela 3. Razões das frações dos hidrocarbonetos aromáticos e saturados para as 26 amostras de óleo analisadas

Hidrocarbonetos aromáticos

\begin{tabular}{|c|c|c|c|c|c|c|c|c|c|c|c|c|c|c|}
\hline \multirow[b]{2}{*}{ Amostra } & \multirow[b]{2}{*}{ Bacia $^{(*)}$} & \multirow{2}{*}{$\begin{array}{l}\text { Fen } \\
\text { IMF }\end{array}$} & \multirow[b]{2}{*}{ RMN } & \multicolumn{3}{|c|}{ Naft } & \multicolumn{2}{|c|}{$\begin{array}{l}\text { 4-MDBT/ } \\
\text { Fen }\end{array}$} & \multicolumn{3}{|c|}{ DBT } & \multicolumn{3}{|c|}{ Hidrocarbonetos saturados } \\
\hline & & & & RDMN & RTMN & RTeMN & RMD & $\begin{array}{c}\text { RMDBT } \\
4 / 1\end{array}$ & $\begin{array}{c}\text { RDMDBT } \\
2,4 / 1,4\end{array}$ & $\begin{array}{c}\text { RDMDBT } \\
4,6 / 1,4\end{array}$ & $\begin{array}{l}\text { RTMDBT } \\
(38 / 40)\end{array}$ & $\mathrm{Pr} / \mathrm{Fi}$ & $\begin{array}{l}\mathrm{C}_{29} \alpha \alpha \alpha \\
\mathrm{S} /(\mathrm{S}+\mathrm{R})\end{array}$ & $\begin{array}{c}\mathrm{C}_{29}(\mathrm{~S}+\mathrm{R}) \\
\alpha \beta \beta /(\alpha \alpha \alpha+\alpha \beta \beta)\end{array}$ \\
\hline CA-99 & $\mathrm{CA}$ & 0,66 & 1,45 & 0,22 & 1,46 & 0,71 & 0,04 & 1,32 & 0,73 & 0,91 & 2,39 & n.d. & 0,47 & 0,39 \\
\hline CA-111 & $\mathrm{CA}$ & n.d. & n.d. & n.d. & n.d. & n.d. & n.d. & n.d. & n.d. & n.d. & n.d. & 1,15 & 0,47 & 0,39 \\
\hline CA-77 & $\mathrm{CA}$ & 0,46 & 1,64 & n.d. & 1,07 & n.d. & 0,04 & 0,55 & 0,63 & 0,56 & 1,44 & 1,56 & 0,58 & 0,39 \\
\hline CA-79 & $\mathrm{CA}$ & 0,78 & 1,73 & 0,29 & 1,61 & 0,69 & 0,05 & 0,76 & 0,83 & 1,05 & 2,84 & 1,57 & 0,57 & 0,47 \\
\hline JE-374 & $\mathrm{JE}$ & 1,25 & 1,56 & 1,39 & 3,59 & 0,78 & 0,29 & 2,91 & 0,80 & 1,09 & 2,88 & 0,98 & 0,24 & 0,31 \\
\hline JE-371 & $\mathrm{JE}$ & 0,98 & 1,55 & 0,32 & 1,15 & 0,81 & 0,22 & 3,41 & 0,88 & 1,46 & 2,39 & 0,82 & 0,28 & 0,46 \\
\hline JE-121 & $\mathrm{JE}$ & 0,63 & 1,10 & 0,90 & 0,61 & 0,63 & 0,23 & 2,71 & 0,47 & 0,43 & 0,97 & 1,64 & 0,52 & 0,55 \\
\hline JE-491 & $\mathrm{JE}$ & 0,54 & 1,30 & 0,73 & 1,08 & 0,48 & 0,45 & 2,04 & 0,18 & 0,59 & 1,47 & 0,84 & 0,38 & 0,49 \\
\hline SE-1 & SE & 0,86 & 0,82 & 0,28 & 0,52 & n.d. & n.d. & n.d. & n.d. & n.d. & n.d. & 0,73 & 0,29 & 0,28 \\
\hline SE-2 & SE & 0,81 & 0,83 & 0,37 & n.d. & 0,46 & n.d. & n.d. & n.d. & n.d. & n.d. & 1,30 & 0,34 & 0,40 \\
\hline SE-6 & SE & 0,68 & 0,58 & 0,15 & n.d. & 0,87 & 0,09 & 7,48 & 0,98 & 1,38 & 3,14 & 1,82 & 0,57 & 0,67 \\
\hline SE-11 & SE & 0,83 & 0,83 & 0,18 & 2,38 & 0,70 & 0,14 & 4,59 & 1,02 & 1,09 & 2,25 & 1,08 & 0,60 & 0,40 \\
\hline SE-95 & SE & 0,54 & 0,79 & 0,25 & n.d. & 0,49 & 0,21 & 3,93 & 0,29 & 0,62 & 0,96 & 2,31 & 0,50 & 0,30 \\
\hline SE-114 & SE & 0,67 & 0,69 & 0,14 & 0,63 & 0,84 & 0,33 & 3,47 & 0,67 & 0,97 & 1,74 & 0,85 & 0,53 & 0,62 \\
\hline SE-73 & SE & 0,55 & 0,94 & 0,26 & 0,78 & n.d. & 0,13 & 2,63 & 0,23 & 0,62 & 1,17 & 1,07 & 0,60 & 0,40 \\
\hline SE-77 & SE & n.d. & 1,27 & 0,26 & 2,05 & 0,78 & 0,11 & 2,03 & 0,43 & 0,60 & 1,65 & 0,11 & 0,50 & 0,40 \\
\hline SE-44 & SE & 1,01 & 0,10 & 0,10 & 0,29 & 0,88 & 0,08 & 12,55 & 0,37 & 1,95 & 4,80 & 2,00 & 0,00 & 0,00 \\
\hline AL-27 & $\mathrm{AL}$ & 0,70 & 0,90 & 0,17 & 1,41 & 0,76 & 0,13 & 5,46 & 0,67 & 0,87 & 1,89 & n.d. & 0,59 & 0,59 \\
\hline AL-1 & $\mathrm{AL}$ & 0,70 & 0,92 & 0,20 & n.d. & n.d. & 0,12 & 1,99 & 0,20 & 0,61 & 1,60 & 1,35 & 0,50 & 0,50 \\
\hline AL-4 & $\mathrm{AL}$ & n.d. & 0,91 & n.d. & n.d. & n.d. & n.d. & n.d. & n.d. & n.d. & n.d. & 0,64 & 0,60 & 0,55 \\
\hline AL-9 & $\mathrm{AL}$ & n.d. & 1,04 & n.d. & n.d. & n.d. & n.d. & n.d. & n.d. & n.d. & n.d. & 2,14 & 0,41 & 0,47 \\
\hline SA-A & SA & 0,85 & 2,20 & n.d. & n.d. & n.d. & 0,37 & 16,68 & 1,32 & 1,91 & 5,73 & 1,50 & 0,61 & 0,61 \\
\hline SA-B & SA & 0,81 & 1,61 & n.d. & n.d. & n.d. & 0,25 & 5,72 & 0,73 & 1,04 & 2,17 & 1,40 & 0,61 & 0,56 \\
\hline SA-5 & SA & 0,79 & 1,25 & 0,31 & 1,02 & n.d. & 0,23 & 5,97 & 0,67 & 0,96 & 1,97 & 1,30 & 0,62 & 0,57 \\
\hline SA-6 & SA & 0,54 & 1,86 & 2,38 & 1,21 & n.d. & 0,04 & 7,30 & 1,05 & 1,30 & 2,94 & 1,50 & 0,59 & 0,55 \\
\hline SA-D & SA & 0,75 & 1,75 & 0,09 & 1,33 & n.d. & 0,10 & 3,31 & 0,58 & 1,02 & 2,50 & 1,40 & 0,50 & 0,46 \\
\hline
\end{tabular}

(*): CA -Camamu-Almada; JE - Jequitinhonha; SE-Sergipe; AL - Alagoas; SA - Santos. Fen.: Fenantreno, Naft.: Naftaleno, 4-MDBT: 4-Metil-dibenzotiofeno, DBT: dibenzotiofeno; n.d.: não detectado.

por exemplo, a razão 4-metil-dibenzotiofeno/1-metil-dibenzotiofeno (RMDBT4/1). ${ }^{11,17}$ Além desta, outras razões também foram utilizadas, dentre as quais 2,4-dimetil-dibenzotiofeno/1,4-dimetil-dibenzotiofeno (2,4-DMDBT/1,4-DMDBT) e 4,6-dimetil-dibenzotiofeno/1,4dimetil-dibenzotiofeno (4,6-DMDBT/1,4-DMDBT), obtidas através do cromatograma de massa $\mathrm{m} / \mathrm{z}, 212$; e, por último, a razão de trimetil-dibenzotiofenos (TMDBT), obtido a partir do cromatograma de massa $m / z, 226$, sendo a razão entre o TMDBT (substância 38)/ TMDBT (substância 40).

Observa-se que a razão do TMDBT (substância 38)/TMDBT (substância 40), referentes aos trimetil-dibenzotiofenos (compostos 38 e 40, Figura 2), tende a aumentar conforme o nível da evolução térmica. ${ }^{18}$ Os valores obtidos dos parâmetros de evolução térmica para as amostras de óleos das bacias estudadas apresentaram distribuições semelhantes nas correlações RMDBT4/1 contra RDMDBT2,4/1,4, RMDBT4/1 contra RDMDBT4,6/1,4 e RMDBT4/1 contra RTMDBT (substâncias 38/40), como ilustrado na Figura 2. Porém, uma amostra da Bacia de Sergipe, SE-44, apresentou um valor maior para a razão RMDBT4/1. Da mesma forma, uma amostra de óleo da Bacia de
Santos, SA-A, também apresentou valor pronunciado para os parâmetros RMDBT4/1 (Figura 2) e RDMDBT2,4/1,4.

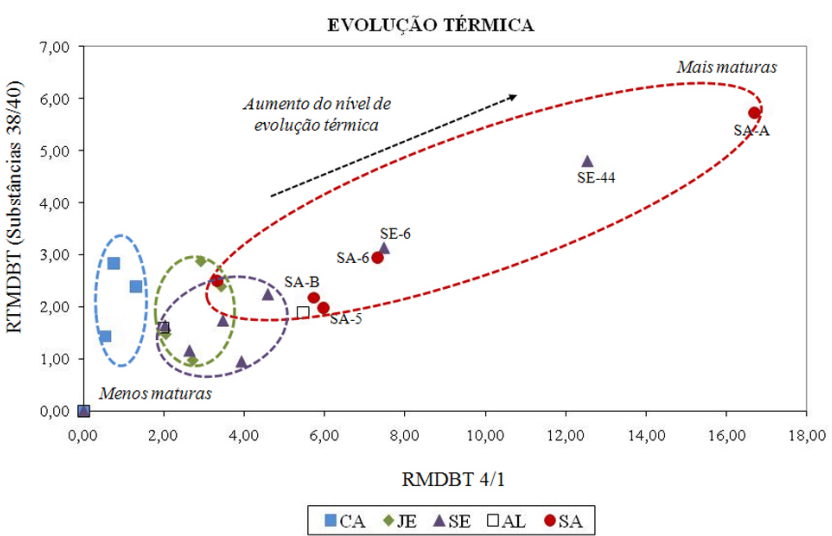

Figura 2. Gráfico de correlação dos parâmetros de maturação RMDBT4/1 contra RTMDBT (38/40), referente às amostras de óleos estudadas 
Tabela 4. Relação dos compostos identificados nas amostras de óleo analisadas

\begin{tabular}{|c|c|}
\hline Compostos identificados & Pico \\
\hline Naftaleno & 1 \\
\hline 2-Metil-naftaleno & 2 \\
\hline 1-Metil-naftaleno & 3 \\
\hline 2,6- + 2,7-Dimetil-naftaleno & 4 \\
\hline 1,3- + 1,7-Dimetil-naftaleno & 5 \\
\hline 1,6-Dimetil-naftaleno & 6 \\
\hline 1,4- + 2,3-Dimetil-naftaleno & 7 \\
\hline 1,5-Dimetil-naftaleno & 8 \\
\hline 1,2-Dimetil-naftaleno & 9 \\
\hline 1,3,7-Trimetil-naftaleno & 10 \\
\hline 1,3,6-Trimetil-naftaleno & 11 \\
\hline 1,3,5- + 1,4,6-Trimetil-naftaleno & 12 \\
\hline 2,3,6-Trimetil-naftaleno & 13 \\
\hline $1,2,7-+1,6,7-+1,2,6$-Trimetil-naftaleno & 14 \\
\hline 1,2,4-Trimetil-naftaleno & 15 \\
\hline 1,2,5-Trimetil-naftaleno & 16 \\
\hline 1,3,5,7-Tetrametil-naftaleno & 17 \\
\hline 1,3,6,7-Tetrametil-naftaleno & 18 \\
\hline 1,2,4,6- + 1,2,4,7- + 1,4,6,7-Tetrametil-naftaleno & 19 \\
\hline 1,2,5,7-Tetrametil-naftaleno & 20 \\
\hline 2,3,6,7-Tetrametil-naftaleno & 21 \\
\hline 1,2,6,7-Tetrametil-naftaleno & 22 \\
\hline 1,2,3,7-Tetrametil-naftaleno & 23 \\
\hline 1,2,3,6-Tetrametil-naftaleno & 24 \\
\hline $1,2,5,6-+1,2,3,5$-Tetrametil-naftaleno & 25 \\
\hline Fenantreno & 26 \\
\hline 3-Metil-fenantreno & 27 \\
\hline 2-Metil-fenantreno & 28 \\
\hline 1-Metil-fenantreno & 29 \\
\hline 9-Metil-fenantreno & 30 \\
\hline 4-Metil-dibenzotiofeno & 31 \\
\hline 1-Metil-dibenzotiofeno & 32 \\
\hline 4,6-Dimetil-dibenzotiofeno & 33 \\
\hline 2,4-Dimetil-dibenzotiofeno & 34 \\
\hline 1,4-Dimetil-dibenzotiofeno & 35 \\
\hline Trimetil-dibenzotiofeno (substância 36) & 36 \\
\hline Trimetil-dibenzotiofeno (substância 37) & 37 \\
\hline Trimetil-dibenzotiofeno (substância 38) & 38 \\
\hline Trimetil-dibenzotiofeno (substância 39) & 39 \\
\hline Trimetil-dibenzotiofeno (substância 40) & 40 \\
\hline Trimetil-dibenzotiofeno (substância 41) & 41 \\
\hline Trimetil-dibenzotiofeno (substância 42) & 42 \\
\hline
\end{tabular}

Neste estudo, o gráfico de correlação RMDBT4/1 contra RTMDBT (38/40) foi o que melhor agrupou as amostras quanto ao parâmetro de maturação, apresentando um coeficiente de correlação de 0,8588 . A correlação dos parâmetros RMDBT4/1 contra RTMDBT (38/40) permitiu um escalonamento das amostras quanto ao grau de evolução térmica possibilitando, portanto, o agrupamento das mesmas com relação à maturidade. De modo geral, as amostras de óleo da Bacia de Santos são as mais maturas (SA-A e SA-6, além de SA-B e SA-5). Este dado está em acordo com o observado através da análise de diamantoides para estas mesmas amostras. ${ }^{13,14,16}$ Para a amostra SE-44, por apresentar elevada maturidade, não foi possível a análise dos parâmetros de maturação utilizando os parâmetros usuais de biomarcadores $\mathrm{S} /(\mathrm{S}+\mathrm{R})$ e $\alpha \beta \beta /(\alpha \alpha \alpha+\alpha \beta \beta)$ esteranos (normalmente obtida utilizando o esterano com 29 átomos de carbono, o etil-colestano; $\alpha \beta \beta \mathrm{C}_{29}=5 \alpha(\mathrm{H}), 14 \beta(\mathrm{H}), 17 \beta(\mathrm{H})$-etil-colestano), pois estes não foram detectados. Tal interpretação mostra-se adequada, pois quanto maior a maturação, maior o craqueamento dos biomarcadores e hidrocarbonetos saturados.

As amostras de óleo da Bacia de Jequitinhonha e Bacia de Camamu foram as que apresentaram os menores valores em média para ambos os parâmetros, caracterizando-as como as menos maturas no grupo de óleos das bacias avaliadas. Estas foram seguidas pelas amostras da Bacia de Santos, as quais apresentaram maiores valores para o parâmetro RMD4-1, assim como para a maioria das amostras da Bacia de Sergipe, mostrando que as mesmas apresentam uma maturação intermediária.

Em trabalhos anteriores com as mesmas amostras da Bacia de Santos, ${ }^{13,14}$ os parâmetros de evolução térmica obtidos através da fração dos hidrocarbonetos saturados como, por exemplo $\mathrm{C}_{29} \alpha \alpha \alpha \mathrm{S} /(\mathrm{S}+\mathrm{R})$ e $\mathrm{C}_{29}(\mathrm{~S}+\mathrm{R}) \alpha \beta \beta /(\alpha \alpha \alpha+\alpha \beta \beta)$, apresentaram resultados indicando níveis de maturidade elevados. Seus valores de equilíbrio atingem a janela de geração do óleo entre 0,52 a 0,55 para $\mathrm{C}_{29} \alpha \alpha \alpha \mathrm{S} /(\mathrm{S}+\mathrm{R})$ e entre 0,67 a 0,71 para $C_{29}(\mathrm{~S}+\mathrm{R}) \alpha \beta \beta /(\alpha \alpha \alpha+\alpha \beta \beta)$, onde se encontram na fase de equilíbrio entre os isômeros. Estas razões são utilizadas para medir a evolução térmica da rocha geradora ao longo da zona imatura e parte da zona principal de geração do óleo ("janela de geração do óleo"). ${ }^{13,14}$ Isto está de acordo com o que foi observado em relação ao parâmetro RMDBT4/1 contra RTMDBT (38/40), também mostrando a elevada maturidade das amostras. Pode-se afirmar, portanto, que os resultados obtidos quanto à evolução térmica das amostras por meio de razões utilizando hidrocarbonetos aromáticos, são muito semelhantes àqueles obtidos por razões de hidrocarbonetos saturados mencionados acima (Figura 3). Para este gráfico, o coeficiente de correlação observado foi de 0,7523 . Isso demonstra a validade do uso dos hidrocarbonetos aromáticos como indicadores de evolução térmica a partir das razões calculadas neste trabalho. Para a avaliação da maturação, os hidrocarbonetos aromáticos apresentaram melhores resultados, pois possuem uma maior resistência ao processo de degradação (térmica e biológica). ${ }^{2,5,12}$

Para amostras que se encontram super maturas, ou seja, craqueadas, os parâmetros de maturação baseados em biomarcadores

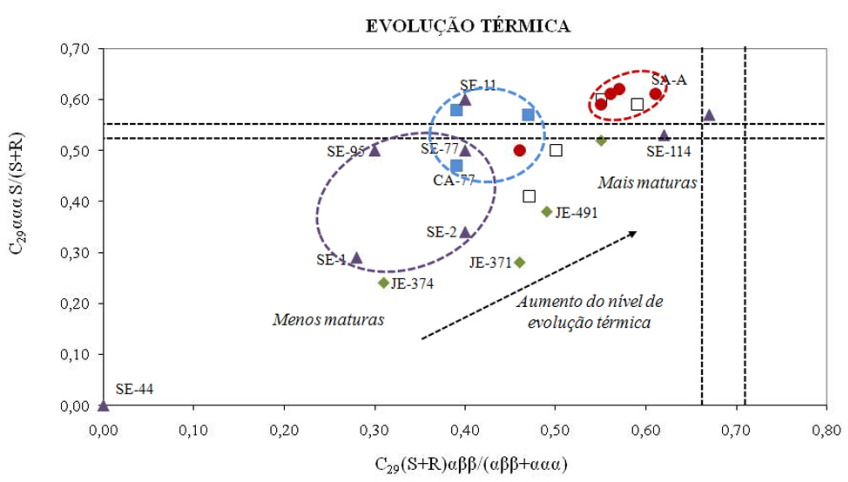

$\because \mathrm{CA} \bullet \mathrm{JE} \triangle \mathrm{SE} \quad \square \mathrm{AL} \bullet \mathrm{SA}$

Figura 3. Gráfico de correlação dos parâmetros de maturação $C_{29} \alpha \alpha \alpha \mathrm{S} /$ $(S+R)$ contra $C_{29}(S+R) \alpha \beta \beta /(\alpha \beta \beta+\alpha \alpha \alpha)$, referente às amostras de óleos estudadas. $\alpha \alpha \alpha C_{29}=5 \alpha(H), 14 \alpha(H), 17 \alpha(H)$-etil-colestano 
saturados como os esteranos ficam alterados, estão fora da faixa de equilíbrio ou, ainda, estes biomarcadores não são mais detectados. Desta forma, os hidrocarbonetos aromáticos, por serem mais resistentes ao processo de craqueamento, podem e devem ser utilizados. Percebe-se que para estes obtêm-se os melhores resultados. Como os diamantoides, os hidrocarbonetos aromáticos são mais resistentes, sendo excelentes parâmetros para avaliação de amostras de elevada maturação e craqueadas.

Por fim, dentre os compostos aromáticos analisados, os dados obtidos através da análise das frações dos compostos aromáticos sulfurados foram os que melhor apresentaram resultados para avaliar a evolução térmica. É importante notar que os valores dos parâmetros de maturação utilizados para correlacionar os óleos são de rochas geradoras de diferentes origens. Isto sugere que a mudança seja similar na distribuição dos compostos aromáticos sulfurados de acordo com o aumento da maturação, das características da litologia e fácies orgânica. ${ }^{18}$

\section{CONCLUSÃO}

A partir da avaliação dos parâmetros de origem utilizando a razão 4-metil-dibenzotiofeno/fenantreno (MDR) com a razão pristano/fitano $(\mathrm{Pr} / \mathrm{Fi})$ concluiu-se que as amostras de óleos das bacias estudadas estão provavelmente associadas com dois ambientes deposicionais distintos: lacustre para as amostras da Bacia do Jequitinhonha e marinho para a maioria das amostras provenientes das bacias de Santos Camamu-Almada, Sergipe e Alagoas.

A correlação dos parâmetros de evolução térmica, utilizando os compostos aromáticos sulfurados nas razões 4-metildibenzotiofeno/1-metil-dibenzotiofeno contra trimetil-dibenzotiofeno (substância 38)/trimetil-dibenzotiofeno (substância 40) [RTMDBT (38/40)], apresentou bons resultados, permitindo o escalonamento das amostras quanto aos diferentes níveis de evolução térmica. Isto permitiu inferir o grau de maturidade para as mesmas, mostrando a maior maturidade para as amostras da Bacia de Santos, níveis intermediários para as amostras das bacias de Sergipe, Alagoas e Jequitinhonha, e os menores níveis para as amostras da Bacia de Camamu-Almada.

A avaliação dos resultados obtidos a partir dos compostos aromáticos sulfurados mostrou que os mesmos são muito úteis na avaliação da evolução térmica da matéria orgânica. Os hidrocarbonetos aromáticos passam a ser uma ferramenta a mais a ser utilizada na avaliação da maturidade de óleos. No entanto, não apresentaram bons resultados quanto à avaliação da origem.

\section{MATERIAL SUPLEMENTAR}

As Figuras 1S e 2S estão disponíveis gratuitamente em http:// quimicanova.sbq.org.br, na forma de arquivo PDF.

\section{AGRADECIMENTOS}

Ao CNPq, à Capes e ANP/PRH-02.

\section{REFERÊNCIAS}

1. Tissot, B. P.; Welte, D. H.; Petroleum Formation and Occurrence, $2^{\text {nd }}$ ed., Springer-Verlag: Berlin, 1984.

2. Peters, K. E.; Walters, C. C.; Moldowan, J. M.; The Biomarker Guide: Biomarker and Isotopes in Petroleum Exploration and Earth History, $2^{\text {nd }}$ ed., Cambridge University: Nova York, 2005, vol. 2.

3. Eglinton, G.; Mar. Geol. 1993, 113, 141.

4. Nabbefeld, B.; Grice, K.; Schimmelmann, A.; Summons, R. E.; Troitzsch, U.; Twitchett, R. J.; Org. Geochem. 2010, 41, 78.

5. Dominguez, Z.; Guzzo, J. V. P.; Azevedo, D. A.; Quim. Nova 2008, 21, 1330.

6. Radke, M.; Willsch, H.; Geochim. Cosmochim. Acta 1994, 58, 5223.

7. van Aarssen, B. G. K.; Hessels, J. K. C.; Abbink, O. A.; Leeuw, J. W.; Geochim. Cosmochim. Acta 1992, 56, 1231.

8. Armstroff, A.; Wilkes, H.; Schwarzbauer, J.; Littke, R; Horsfield, B.; Org Geochem. 2007, 38, 1692.

9. Smith, J. W.; George, S. C.; Batts, B. D.; APEA J. 1994, 34, 504

10. Budzinski, H.; Garrigues, P.; Connan, J.; Devillers, J.; Domine, D.; Radke, M.; Oudin, J. L.; Geochim. Cosmochim. Acta 1995, 59, 2043.

11. Radke, M.; Welte, D. H.; Willsch, H.; Geochim. Cosmochim. Acta 1986, 64, 275.

12. Sardiñas, Z. D.; Tese de Doutorado, Universidade Federal do Rio de Janeiro, Brasil, 2008.

13. Jesuino, L. S.; Dissertação de Mestrado, Universidade Federal do Rio de Janeiro, Brasil, 2005; Tamanqueira, J. B.; Dissertação de Mestrado, Universidade Federal do Rio de Janeiro, Brasil, 2006; Wiedemann, L. S. M.; Tese de Doutorado, Universidade Federal do Rio de Janeiro, Brasil, 2006; Araujo, F. C.; Dissertação de Mestrado, Universidade Federal do Rio de Janeiro, Brasil, 2007.

14. Springer, M. V.; Garcia, D. F.; Gonçalvez, F. T. T.; Landau, L.; Azevedo, D. A.; Org. Geochem. 2010, 41, 1013; Azevedo, D. A.; Silva, T. F.; Silva, D. B.; Quim. Nova 2008, 32, 1770; Azevedo, D. A.; Tamanqueira, J. B.; Dias, J. C. M.; Carmo, A. P. B.; Landau, L.; Gonçalvez, F. T. T.; Fuel 2008, 87, 2122.

15. Radke, M.; Mar. Petrol. Geol. 1988, 5, 224.

16. Sivan, P.; Datta, G. C.; Singh, R. R.; Org Geochem. 2008, 39, 1620.

17. Huang, H.; Bowler, B. F. J.; Oldenburg, T. B. P.; Larter, S. R.; Org. Geochem. 2004, 35, 1619.

18. Chakhmakhchev, A.; Suzuki, M.; Takayama, K.; Org. Geochem. 1997, 26,483 . 
AVALIAÇÃO GEOQUÍMICA DE ÓLEOS BRASILEIROS COM ÊNFASE NOS HIDROCARBONETOS AROMÁTICOS

\section{Jean Romei Heckmann* e Luiz Landau}

COPPE - Instituto Alberto Luiz Coimbra de Pós-graduação e Pesquisa de Engenharia, Universidade Federal do Rio de Janeiro, 21941-909 Rio de Janeiro - RJ, Brasil

Félix Thadeu Teixeira Gonçalves

Vale Óleo e Gás, 20020-900 Rio de Janeiro - RJ, Brasil

Ricardo Pereira e Débora de Almeida Azevedo

Instituto de Química, Universidade Federal do Rio de Janeiro, 21941-909 Rio de Janeiro - RJ, Brasil
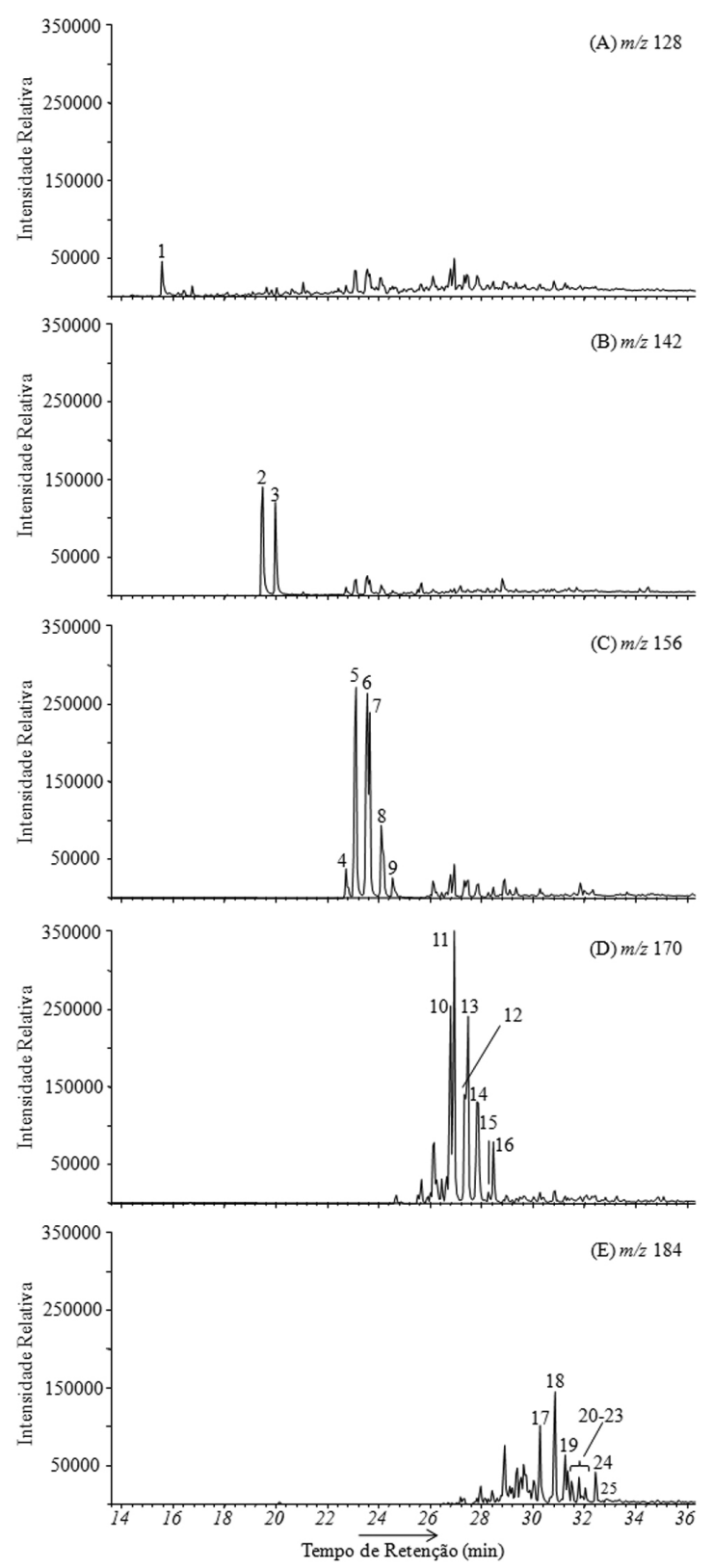

Figura 1S. Cromatogramas de massas dos íons: (A) $\mathrm{m} / \mathrm{z}$ 128, característico do naftaleno, (B) $\mathrm{m} / \mathrm{z}$ 142, característico dos metil-naftalenos, (C) $\mathrm{m} / \mathrm{z}$ 156, característico dos dimetil-naftalenos, (D) $\mathrm{m} / \mathrm{z}, 170$, característico dos trimetil-naftalenos e (E) $\mathrm{m} / z$. 184, característico dos tetrametil-naftalenos, demonstrando a distribuição destes, para a amostra JE-371. A identificação dos picos é apresentada na Tabela 4

*e-mail: jean.heckmann@hotmail.com 

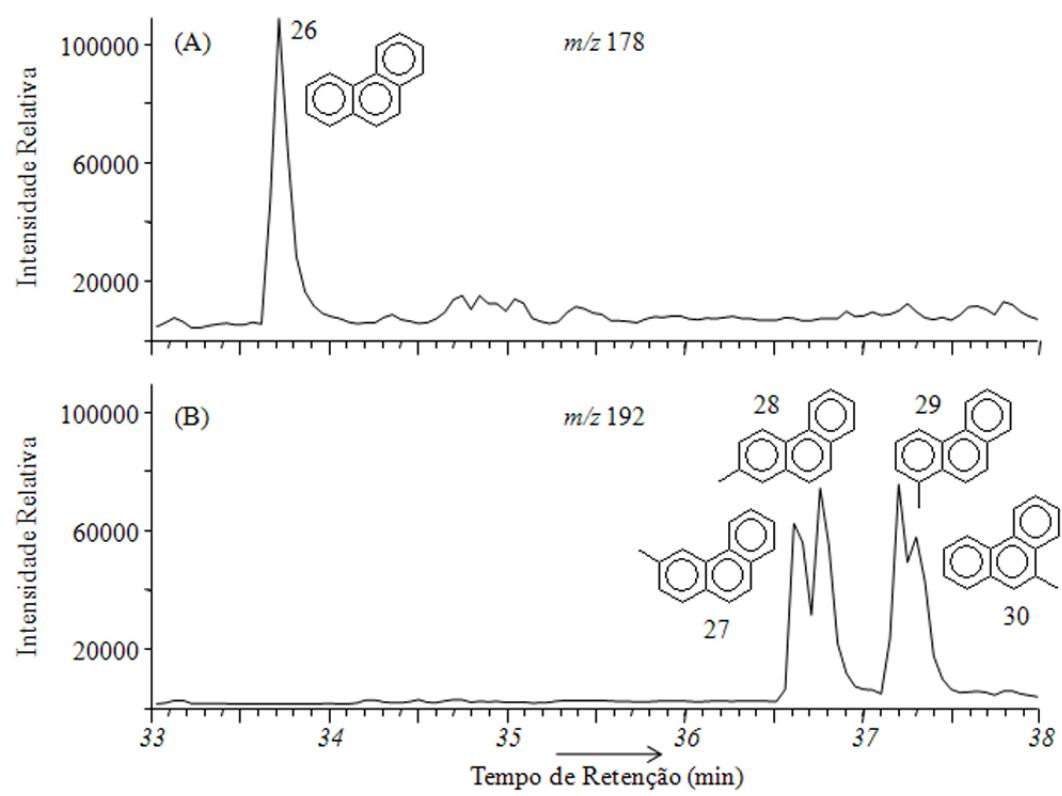

Figura 2S. Cromatograma de massas dos íons: (A) $\mathrm{m} / \mathrm{z}, 178$, característico do fenantreno e (B) $\mathrm{m} / \mathrm{z}, 192$, característico dos metil-fenantrenos, demonstrando a distribuição destes, para a amostra SE-2. A identificação dos picos é apresentada na Tabela 4 